\title{
Zu spät, Du rettest die Schwester nicht mehr*
}

\author{
Glosse mit einigen zusätzlichen Betrachtungen
}

K. Bösch

* sehr frei nach Schiller.

Korrespondenz:

Dr. med. Kurt Bösch

Schlossbach

Postfach

CH-9404 Rorschacherberg

E-Mail: kuboesch@paus.ch
«He Sie!» oder, stellvertretend, «Hallo Sie!»

Nicht ungebräuchlicher und meist wirksamer, wenn auch nicht sehr gediegener Ruf, zum Beispiel auf der Strasse, auf der Baustelle, im Gedränge, aber wohl kaum im Spitalbett und auch nicht im Restaurant. Dort mag der Vertraute nach dem Rösli oder der Nadia rufen, wenn er sich vertraut genug glaubt.

Auch «Frau!» oder «Hallo Frau!» als Ersatz für «He Sie» findet nicht überall Anklang und will sich nicht durchsetzen. "Frau Huber», «Frau Hürlimann» lässt sich auch - entsprechende Kenntnisse vorausgesetzt - rufen.

Der Engländer ruft Miss, nicht ganz unzweckmässig. Wer damit gemeint ist, entscheiden die jeweils Anwesenden. Und die Miss ist ja eigentlich das bei uns verpönte Fräulein.

«Hallo!» Ja warum eigentlich nicht einfach «Hallo!»?

Ich spreche von den (nicht mehr ganz neuen, aber immer noch aktuellen) Schwierigkeiten als Patient, unmissverständlich eine Pflegeperson zu sich zu rufen. Sie ist heutzutage in den meisten Häusern nun nicht mehr «Schwester Susi» oder «Schwester Paula» sondern Frau Huber oder Frau Müller und unterscheidet sich damit weder von der Bettnachbarin, noch vom Abteilungsmädchen, noch von der vornehmen Hotelfachangestellten, noch von Besuchern gleichen Namens. Sie strömt auch nicht mehr das Odium der Hilfreichen aus. Das hat sie sich selber eingebrockt, sie hat es so wollen. Oder vielmehr die Ideologinnen der Zunft haben es so gewollt. Wieso eigentlich? Das vermag mir niemand bindend zu erklären, ich komme darauf zurück. Das Namensschild oder der Batch helfen auch nicht weiter, wenn sie nicht getragen werden oder schwer zu lesen sind.

Inzwischen ist ja die Krankenschwester zur Pflegefachfrau geworden, einige sind sogar Doktorinnen der Pflegewissenschaften.

Grüezi, Frau Dokterschwöschter oder Schwöschterdokter Ruth Hueber? Nein, das geht wohl nicht, auch sind auf den Pflegestationen die Akademikerinnen noch nicht zu finden. Was diese dereinst, wenn sie vielleicht gehäuft auftreten, für mich, den Patienten, noch tun wollen, für welche Verrichtungen sie noch bereit sein werden, bleibt offen. Die Tendenz, den Patienten selbst für vieles als zuständig zu erklären (mündig, wie man gemäss modernen Pflegemodellen sagt) und ihm unerwartete Aufgaben zuzuschieben, läuft zur sprachlichen Entwicklung parallel. So oder so, mit «Doktor» würden sie, die studierten Pflegerinnen, kaum begrüsst werden. Auch der richtige Doktor kann heute nur noch selten damit rechnen, als Doktor angesprochen zu werden, auch wenn seine Berufsbezeichnung und sein akademischer Grad gleich lauten. Es tönt anscheinend zu sehr nach Ehrerbietung. Ich gestehe gerne und naiv ein das ganz nebenbei -, dass ich mich über diese verstaubte Anrede jedesmal freue, wenn man mir damit auf der Strasse, bei der Kasse im Supermarkt, im Labor bei der Quickbestimmung, in der Apotheke, beim Bäcker oder wo und wann auch immer fröhlich und laut begegnet. Das ist ohne Zweifel eine Alterserscheinung.

Nun, zu was für einer Rufform man sich als Spitalpatient auch immer entschliessen mag, nach einigen wenigen Stunden und zwanzigmal in der Woche heisst die Zuständige eh wieder ganz anders, und eine für alle gültige, hilfemobilisierende und unverwechselbare Bezeichnung existiert nicht (mehr). Ein ungelöstes Problem, man mag es als nebensächlich empfinden, aber in gewissen Fällen gelangt es sogar zu lebenswichtiger Bedeutung. Aber irgendwie wird es schon klappen, wir mussten uns ja auch beim gastgewerblichen Fachpersonal nach der Abschaffung des Fräuleins zu helfen versuchen.

Nun aber: Wie konnte es dazu kommen, dass man die Krankenschwester nicht mehr Schwester nennen und rufen darf? Möglicherweise begann das, wenn man es so sehen will, vor 40 bis 60 Jahren mit dem nie schlüssig begründeten Verschwinden der «Schwesternhäubchen», auch der adretten Varianten und der Schwesterntrachten, auch der kleidsamen Exemplare.

Ja, die barmherzigen Schwestern haben ausgespielt, fast. Ihre Barmherzigkeit ist sogar mit Schuld an der Ausmerzaktion der Bezeichnung. Emanzipatorische Überlegungen $\mathrm{zu}$ falschverstandenen Gegebenheiten, die vor allem die Ideologinnen anstellen, führten dazu. Feministische Scharfmacherinnen sind in den oberen Etagen gar nicht so selten zu finden. Die Schwester wurde - historisch zwar korrekt - mit Klosterfrauen assoziiert, mit schwarz gekleideten, überdies (abgesehen von den Diakonissen) 
katholischen Gestalten, Irrwischen. In den Augen und Ohren der Ideologinnen wohnen diesen Gestalten Zeichen von Unterwürfigkeit, von fehlender Kompetenz und Eigenständigkeit, von mangelnder Professionalität inne oder, moderner ausgedrückt, es tönt, oh Graus, nach Dienstleistung, nach Service. Das grosse Heer der Patienten, der «Serviceabhängigen» und des breiten Volkes empfindet definitiv nicht so. Die Hilfeleistung wird natürlich erwartet, aber als solche hoch geschätzt und dankbar empfangen. Mit der ihm neuerdings zugemuteten Mündigkeit (auch Empowerment genannt) und der ihm abverlangten Selbständigkeit wird der Patient oft nicht fertig, sie ist ihm in seiner Hilflosigkeit kaum ein Anliegen.

Nebenbei bemerkt: Klosterfrauen und Diakonissen waren der damaligen Zeit entsprechend nicht im geringsten unprofessionell, wie man ihnen diskriminierend unterschieben will, nur waren Empowerment und Mündigkeitserklärung der Patienten noch kein Thema. Aber: Dafür waren die «Schwestern» auf vielen Gebieten sehr eigenständig, sehr erfahren und in den letzten Jahrzehnten auch schulmässig gründlich ausgebildet. Eine «staatl. dipl. zertif. Barmherzigkeitsfachangestellte» gab's freilich noch nicht. Es war klar und selbstverständlich, dass der Dienst am Patienten gemeinsam mit den Medizinern an erster Stelle zu stehen hatte. Er war eherner Gehalt des Berufes und der Berufung und der genannte Patient rechnete damit.

$\mathrm{Ob}$ unser Erklärungsversuch nun zutreffen mag oder nicht, die Schwester wurde (wie seinerzeit das Fräulein) im Zuge der Selbstverwirklichung ersatzlos gestrichen. Das damit verbundene Problemchen blieb ungelöst, es wurde sozusagen überwunden. Sie haben es mitbekommen, mit der Umtaufe der Schwester in Pflegefachfrau. Das tönt zwar eckig und abweisend und ist für Hilfeschreie schon gar nicht brauchbar, aber was soll's.

Ein Trost: Wenn man sich umhört, vernimmt man allenthalben, dass verhältnismässig viele diplomierte Krankenschwestern sich selbstbewusst nach wie vor und gerne und ohne Beeinträchtigungsideen, ja sogar nicht ohne Stolz Schwester nennen und/oder nennen lassen, unbehelligt oder sogar ermuntert von ihren Vorgesetzten.

Warum ich diese Zeilen geschrieben habe? Nehmen Sie's als Nachruf.

Es bietet sich in diesem Zusammenhang die Gelegenheit, einige Überlegungen, fragmentarische Beobachtungen und wenig geordnete Gedanken zum Thema anzuführen: Man bemerkt z.B.,
- dass die Wertschätzung, das Ansehen und der Stellenwert, die bzw. den die Pflegeberufe geniessen, sehr hoch war und ist, weit höher als die Ideologinnen unter den Pflegewissenschaftlerinnen uns glauben machen wollen;

- dass dieses Ansehen mit der heraufbeschworenen sogenannten Professionalisierung und mit Bestrebungen zu vermehrter Eigenständigkeit und Abgrenzung nicht verbessert werden kann;

- dass nämlich die Professionalität schon früher da war und geschätzt, aber nicht so genannt wurde.

Man vernimmt ferner und darf glauben,

- dass die Akademisierung, die Intellektualisierung und damit das Abgleiten vom Praktischen ins Theoretische nicht dazu beiträgt, den Pflegeberuf für potentielle Anwärterinnen attraktiver (wie das z.B. der Bodenseerat erhofft) zu machen und die Zuwendung der Pflegenden zum Patienten zu vergrössern. Zum Teil seit Bologna 1999, aber auch sonst einem Trend folgend, versuchen zurzeit auch andere Berufsgruppen diesen Weg. Es geht weg von der Gewerbeschule über die Berufsschule und Berufsmaturität zur Fachschule und darüber hinaus zur Fachhochschule zum Baccalaureus und zum Master. Übrigens hat man offenbar, wenn ich mich nicht täusche, bei der Gleichschaltung mit dem angelsächsischen Studiensystem übersehen, dass keine weibliche Form für Bachelor und Master existiert. Unbegreiflich, dass so etwas passieren durfte;

- dass man, um das hohe Ansehen der Pflegeberufe und den Zuspruch dazu nicht zu schmälern, recht vorsichtig sein sollte mit wenig durchdachten, ideologisch gefärbten Experimenten. Man möchte ja berechtigterweise mehr Lohn, dazu braucht es Gerechtigkeit, Gleichberechtigung, aber auch das Wohlwollen der Bevölkerung und der gegenwärtigen und der zukünftigen Patienten und nicht zuletzt der Geldgeber.

Und zum Schluss: Man beobachtet, dass die erwähnten Probleme grosse Teile der Ärzteschaft offensichtlich nur am Rand oder überhaupt nicht berühren und dass die Kenntnis der herrschenden Entwicklung oft sogar bei Klinikvorstehern fast fehlt. Es kommt wohl nicht von ungefähr, dass man dieser Thematik in der Ärztezeitung nicht begegnet. Man liest weder von den neuen Pflegemodellen, von Höfa, noch von Pflegeanamnese und Pflegevisite usw. Man kann auch vermuten, dass verhältnissmässig 
wenig Ärzte wissen, dass heute eine Grundausbildung für Gesundheitsfachangestellte angeboten wird. Sie dürften auch deren Curriculum nicht kennen und nicht wissen, dass diese, trotz ihres etwas zwittrigen Wesens - glücklicherweise? - grossen Zuspruch findet. Was alles kaum mehr etwas mit dem eingangs aufgeworfenen Problem zu tun hat. Oder vielleicht doch?

\title{
Rollenbilder
}

\author{
Erhard Taverna
}

Die historische Entwicklung der Medizin ist weit mehr als Wissenschaftsgeschichte. $\mathrm{Zu}$ einem bedeutenden Teil ist sie auch eine Sozialgeschichte von Mann und Frau. Wenn Umfragen ergeben, dass die Bevölkerung für mehr «care» statt «cure» votiert, weil Ärzten und Ärztinnen ungenügende soziale Kompetenzen vorgeworfen werden, tönt das wie ein ironischer Abgesang auf überholte Rollenbilder. Der Verstand war bisher immer männlich und das Herz notgedrungen weiblich. Den armen Medizinstudenten werden professionelle Ethiker, Soziologen und Kommunikationswissenschaftler das Defizit an «Humanities» auskurieren. Inzwischen hat sich der «Hilfs- und Tugendberuf für ehrbare Frauen im Dienste der Ärzte» über die Pflegefachfrau zur klinischen Pflegeexpertise (advanced nursing practice) weiterentwickelt.

Die Teilnehmer der WHO-Ministerkonferenz für Pflege- und Hebammenwesen in Europa haben im Juni 2000 ihre Ziele in einer «Erklärung von München» formuliert. Die Behörden sind darin aufgerufen, sechs Millionen Berufstätige durch finanzielle Anreize, vielfältigere Laufbahnmöglichkeiten und neue Tätigkeitsgebiete gegenüber der ärztlichen Dominanz besserzustellen. Im gleichen Jahr wollte in der Schweiz eine nationalrätliche Motion die Pflegeberufe durch gesetzliche Massnahmen aufwerten. Ausschlaggebend war die Sorge über den zunehmenden Personalmangel und die dadurch gefährdete Pflegesicherheit und Pflegequalität. Nachdem Ständerat und Bundesrat die Motion ablehnten, wird seither die Lobbyarbeit im Rahmen der KVG-Revision intensiviert.

Im Jahre 2002 waren in der Schweiz 90\% von 150000 Pflegenden Frauen, von denen 58\% in Teilzeit arbeiteten. Die grosse Mehrheit war angestellt, nur etwa 660 Personen waren freiberuflich tätig. Nur durch aggressive Rekrutierungskampagnen innerhalb und ausserhalb der EU gelingt es grossen Kliniken, die Nachfrage durch einen steigenden Anteil von Ausländerinnen knapp abzudecken. Was bei Ärztinnen und Ärzten beobachtet wird, wiederholt sich auf der Pflegeseite: ökonomische Fragmentierung der Arbeitskräfte, ungebrochene Spezialisierung, berufliche Hierarchisierung und ein Auseinanderdriften der unterschiedlichen Berufsinteressen.

Seit Herbst 2000 bietet die Universität Basel einen Lehrgang für klinisch ausgerichtete Pflegewissenschaft an. Nach angelsächsischem Vorbild werden spezifische Krankheitsprobleme systemisch angegangen und Lösungen für viele Patientengruppen ausgearbeitet. Spezialisierte Pflegeteams übernehmen Betreuungsaufgaben aus der Grundversorgung, wenn nötig flankiert von medizinischen Call Centern. Mit dem neuen Rollenverständnis werden auch die Aufgaben und Grenzen bisher rein ärztlich verwalteter Domänen neu definiert.

Parallel zu den Reformen des Medizinstudiums entstehen im Gesundheitswesen immer neue Lehrgänge, Berufsbilder und Weiterbildungszentren für Pflegende, Mediziner, Ökonomen, Psychologen, Soziologen und Juristen. Neue Gebiete wie Management, Pflegeforschung, Berufspädagogik und Informatik absorbieren die Abgänger der Mittel- und Fachhochschulen. Mit der gegenseitigen Anerkennung von Diplomen und Berufscurricula geschieht ein Umbau des ganzen Ausbildungssystems. Wer ein SRK-anerkanntes Diplom in Pflege oder Geburtshilfe vorweisen kann oder an einer Berufsschule unterrichtet, hat die Qual der Wahl. Eine höhere Fachausbildung in Pflege Stufe I oder II, einen 3jährigen Teilzeitlehrgang für den «Master in Nursing Science» oder eine vertiefte Ausbildung für den Lehrberuf. Die Akademisierung verschärft das Personalproblem an der Basis. Durch die Senkung des Eintrittsalters soll vermehrt Nachwuchs rekrutiert werden. Mit dem neuen Bundesgesetz über die Berufsbildung ist ein neuer Beruf auf Sekundarstufe II entstanden, welcher zum Fähigkeitszeugnis «Fachangestellte Gesundheit» führt. Die Karrierewege sind vielfältig und kaum mehr übersehbar. Gefragt sind neue Umgangsformen zwischen den neuen Akteuren unter sich und mit Patientinnen und Patienten, die auch nicht mehr sind, was sie einmal waren. Vor dem Hintergrund schwindender Finanzen und verschärfter Brotkämpfe dürfte die gegenseitige Anrede das kleinste Problem sein. 